\title{
Neutrino mass anarchy and the Universe
}

\author{
Xiaochuan $\mathbf{L u}^{a, b}$ and Hitoshi Murayama ${ }^{a, b, c}$ \\ ${ }^{a}$ Department of Physics, University of California, \\ Berkeley, California 94720, U.S.A. \\ ${ }^{b}$ Theoretical Physics Group, Lawrence Berkeley National Laboratory, \\ Berkeley, California 94720, U.S.A. \\ ${ }^{c}$ Kavli Institute for the Physics and Mathematics of the Universe (WPI), \\ Todai Institutes for Advanced Study, University of Tokyo, \\ Kashiwa 277-8583, Japan \\ E-mail: luxiaochuan123456@berkeley .edu, hitoshi@berkeley . edu
}

ABSTRACT: We study the consequence of the neutrino mass anarchy on cosmology, in particular the total mass of neutrinos and baryon asymmetry through leptogenesis. We require independence of measure in each mass matrix elements in addition to the basis independence, which uniquely picks the Gaussian measure. A simple approximate $\mathrm{U}(1)$ flavor symmetry makes leptogenesis highly successful. Correlations between the baryon asymmetry and the light-neutrino quantities are investigated. We also discuss possible implications of recently suggested large total mass of neutrinos by the SDSS/BOSS data.

Keywords: Beyond Standard Model, Cosmology of Theories beyond the SM, Neutrino Physics, Statistical Methods

ARXIV EPRINT: 1405.0547 


\section{Contents}

1 Introduction 1

2 Sampling model $\quad 3$

2.1 Gaussian measure 3

2.2 Approximate U(1) flavor symmetry 5

3 Consequences $\quad 6$

3.1 Light-neutrino mixings and mass splitting ratio $\quad 7$

3.2 Mass hierarchy, $m_{\text {eff }}$ and $m_{\text {total }} \quad 8$

$\begin{array}{lll}3.3 & \text { Leptogenesis } & 10\end{array}$

4 Correlations between $\eta_{B 0}$ and light-neutrino parameters $\quad 15$

$5 \quad$ Possible consequences of a large $m_{\text {total }} \quad 17$

6 Conclusions 18

A Basis independence and free entry independence uniquely lead us to Gaussian measure

\section{Introduction}

Neutrino physics is a unique area in particle physics that has many direct consequences on the evolution history and the current state of Universe. It was one of the first hypotheses for the non-baryonic dark matter. Excluding this possibility relied on rather surprising constraint that the density of neutrinos would exceed that allowed by Fermi degeneracy in the core of dwarf galaxies! [1] Because of the free streaming, massive neutrinos would also suppress the large-scale structure, which is still subject to active research. The explosion mechanism of supernova is tied to properties of neutrinos, and hence the chemical evolution of galaxies depend on neutrinos. The number of neutrinos is relevant to Big-Bang Nucleosynthesis. In addition, neutrinos may well have created the baryon asymmetry of the Universe [2] or create the Universe itself with scalar neutrino playing the role of the inflaton $[3,4]$.

Many of the consequences of neutrino properties on the Universe rely on the mass of neutrinos. After many decades of searches, neutrino mass was discovered in 1998 in disappearance of atmospheric neutrinos by the Super-Kamiokande collaboration [5]. Subsequently the SNO experiment demonstrated transmutation of solar electron neutrinos to other active neutrino species [6] corroborated by reactor neutrino data from KamLAND [7]. 
Most recently, the last remaining mixing angle was discovered by the Daya Bay reactor neutrino experiment [8]. Other experiments confirmed this discovery $[9,10]$.

On the other hand, fermion masses and mixings have been a great puzzle in particle physics ever since the discovery of muon. Through decades of intensive studies, we have discovered the existence of three generations and a bizarre mass spectrum and mixings among them. The underlying mechanism for this pattern is still not clear. But the hierarchical masses and small mixings exhibited by quarks and charged leptons seem to suggest that mass matrices are organized by some yet-unknown symmetry principles. The discovery of neutrino masses and mixings seem to even complicate the puzzle. From the current data [11]

$$
\begin{aligned}
\Delta m_{12}^{2} & =(7.50 \pm 0.20) \times 10^{-5} \mathrm{eV}^{2}, \\
\left|\Delta m_{23}^{2}\right| & =\left(2.32_{-0.08}^{+0.12}\right) \times 10^{-3} \mathrm{eV}^{2}, \\
\sin ^{2} 2 \theta_{23} & >0.95(90 \% \text { C.L. }), \\
\sin ^{2} 2 \theta_{12} & =0.857 \pm 0.024, \\
\sin ^{2} 2 \theta_{13} & =0.095 \pm 0.010,
\end{aligned}
$$

the neutrinos also display a small hierarchy $\Delta m_{12}^{2} /\left|\Delta m_{23}^{2}\right| \sim \frac{1}{30},{ }^{1}$ which is quite mild compared to quarks and charged leptons. In addition, unlike quarks and charged leptons, the neutrinos have both large and small mixing angles. Many attempts were made to explain this picture using ordered, highly structured neutrino mass matrices, especially when $\theta_{13}$ was consistent with zero [12-16].

Quite counterintuitively, however, it was pointed out that the pattern of neutrino masses and mixings can also be well accounted for by structureless mass matrices [17]. Mass matrices with independently random entries can naturally produce the small hierarchical mass spectrum and the large mixing angles. This provides us with an alternative point of view: instead of contrived models for the mass matrix, one can simply take the random mass matrix as a low energy effective theory [18]. This anarchy approach is actually more naturally expected, because after all, the three generations possess the exact same gauge quantum numbers. From the viewpoint of anarchy, however, the mass spectrum with large hierarchy and small mixings exhibited by quarks and charged leptons need an explanation. It turns out that introducing an approximate $\mathrm{U}(1)$ flavor symmetry [19, 20] can solve this problem well [18]. This combination of random mass matrix and approximate U(1) flavor symmetry has formed a new anarchy-hierarchy approach to fermion masses and mixings [18].

To be consistent with the spirit of anarchy, the measure to generate the random mass matrices has to be basis independent [18]. This requires that the measure over the unitary matrices be Haar measure, which unambiguously determines the distributions of the mixing angles and CP phases. Consistency checks of the predicted probability distributions of neutrino mixing angles against the experimental data were also performed in great

\footnotetext{
${ }^{1}$ Throughout this paper, we use small hierarchy for a mass spectrum typically like $1: 3: 10$, and large hierarchy for a mass spectrum typically like $1: 10^{2}: 10^{4}$. So in our wording, the neutrinos exhibit a small hierarchy, while the quarks and leptons exhibit a large hierarchy
} 
detail [21, 22]. Although quite successful already, this anarchy-hierarchy approach obviously has one missing brick: a choice of measure to generate the eigenvalues of the random mass matrices. With the only restriction being basis independence, one can still choose any measure for the diagonal matrices at will. However, as we will show in this paper, if in addition to basis independence, one also wants the matrix elements to be distributed independent from each other, then the only choice is the Gaussian measure.

In this paper, we focus on the Gaussian measure to investigate the consequences. As pointed out in [18], the quantities most sensitive to this choice would be those closely related to neutrino masses. We study a few such quantities of general interest, including the effective mass of neutrinoless double beta decay $m_{\text {eff }}$, the neutrino total mass $m_{\text {total }}$, and the baryon asymmetry $\eta_{B 0}$ obtained through a standard leptogenesis [23, 24]. We also present a correlation analysis between $\eta_{B 0}$ and light-neutrino parameters. Recently, the correlation between leptogenesis and light-neutrino quantities was also studied by taking linear measure in [25]. Their results are in broad qualitative agreement with ours in this paper.

The rest of this paper is organized as following. We first motivate our sampling model - Gaussian measure combined with approximate U(1) flavor symmetry — in section 2. Then the consequences of this sampling model is presented in section 3. We show our Monte Carlo predictions on light-neutrino mass hierarchy, effective mass of neutrinoless double beta decay, light-neutrino total mass, and baryon asymmetry through leptogenesis. In section 4, we investigate the correlations between baryon asymmetry and light-neutrino quantities. A recent Baryon Oscillation Spectroscopic Survey (BOSS) analysis suggests that the total neutrino mass could be quite large [26]. Our section 5 is devoted to discuss the consequence of this suggestion. We conclude in section 6 .

\section{Sampling model}

\subsection{Gaussian measure}

To accommodate the neutrino masses, we consider the standard model with an addition of three generations of right-handed neutrinos $\nu_{R}$, which are singlets under $\mathrm{SU}(2)_{L} \times \mathrm{U}(1)_{Y}$ gauge transformations. Then there are two neutrino mass matrices, the Majorana mass matrix $m_{R}$ and the Dirac mass matrix $m_{D}$, allowed by gauge invariance

$$
\begin{gathered}
\Delta \mathcal{L} \supset-\epsilon^{a b} \bar{L}_{a} H_{b}^{\dagger} y_{\nu} \nu_{R}-\frac{1}{2} \bar{\nu}_{R}^{c} m_{R} \nu_{R}+\text { h.c. } \\
\supset-\bar{\nu}_{L} m_{D} \nu_{R}-\frac{1}{2} \bar{\nu}_{R}^{c} m_{R} \nu_{R}+\text { h.c. }
\end{gathered}
$$

where $y_{\nu}=\frac{\sqrt{2}}{v} m_{D}$, with $v=246 \mathrm{GeV}$. With the spirit of anarchy, we should not discard either of them without any special reason. Both should be considered as random inputs. We parameterize them as

$$
\begin{aligned}
& m_{R}=\mathcal{M} \cdot U_{R} D_{R} U_{R}^{T}, \\
& m_{D}=\mathcal{D} \cdot U_{1} D_{0} U_{2}^{\dagger},
\end{aligned}
$$


where $D_{R}$ and $D_{0}$ are real diagonal matrices with non-negative elements; $U_{R}, U_{1}$ and $U_{2}$ are unitary matrices; $\mathcal{M}$ and $\mathcal{D}$ are overall scales.

At this point, the requirement of neutrino basis independence turns out to be very powerful. It requires that the whole measure of the mass matrix factorizes into that of the unitary matrices and diagonal matrices [18]:

$$
\begin{aligned}
d m_{R} & \sim d U_{R} d D_{R}, \\
d m_{D} & \sim d U_{1} d U_{2} d D_{0} .
\end{aligned}
$$

Furthermore, it also demands the measure of $U_{R}, U_{1}$ and $U_{2}$ to be the Haar measure of $\mathrm{U}(3)$ group [18]:

$$
U=e^{i \eta} e^{i \phi_{1} \lambda_{3}+i \phi_{2} \lambda_{8}}\left(\begin{array}{ccc}
1 & 0 & 0 \\
0 & c_{23} & s_{23} \\
0 & -s_{23} & c_{23}
\end{array}\right) \times\left(\begin{array}{ccc}
c_{13} & 0 & s_{13} e^{-i \delta} \\
0 & 1 & 0 \\
-s_{13} e^{i \delta} & 0 & c_{13}
\end{array}\right) \times\left(\begin{array}{ccc}
c_{12} & s_{12} & 0 \\
-s_{12} & c_{12} & 0 \\
0 & 0 & 1
\end{array}\right) e^{i \chi_{1} \lambda_{3}+i \chi_{2} \lambda_{8}},
$$

$d U=d s_{23}^{2} d c_{13}^{4} d s_{12}^{2} d \delta \cdot d \chi_{1} d \chi_{2} \cdot d \eta d \phi_{1} d \phi_{2}$

where $\lambda_{3}=\operatorname{diag}(1,-1,0), \lambda_{8}=\operatorname{diag}(1,1,-2) / \sqrt{3}$, and $c_{12}=\cos \theta_{12}$, etc.

Although basis independence is very constraining, it does not uniquely fix the measure choice of $m_{R}$ or $m_{D}$, because arbitrary measure of the diagonal matrices $D_{R}$ and $D_{0}$ is still allowed. Now let us look at the entries of $m_{R}$ and $m_{D}$. There are 9 complex free entries for $m_{D}$ and 6 complex free entries for the symmetric matrix $m_{R}=m_{R}^{T}$. Generating each free entry independently is probably the most intuitive way of getting a random matrix. However, if one combines this free entry independence with the basis independence requirement, then it turns out there is only one option left - the Gaussian measure:

$$
\begin{aligned}
& d m_{D} \sim \prod_{i j} e^{-\left|m_{D, i j}\right|^{2}} d m_{D, i j}=\left(\prod_{i j} d m_{D, i j}\right) e^{-t r\left(m_{D} m_{D}^{\dagger}\right)} \\
& d m_{R} \sim \prod_{i} e^{-\left|m_{R, i i}\right|^{2}} d m_{R, i i} \prod_{i<j} e^{-2\left|m_{R, i j}\right|^{2}} d m_{R, i j}=\left(\prod_{i \leq j} d m_{R, i j}\right) e^{-\operatorname{tr}\left(m_{R} m_{R}^{\dagger}\right)} .
\end{aligned}
$$

Although this is a known result in random matrix theory [27, 28], we also present our own proof in the appendix.

On one hand, basis independence is necessary from the spirit of anarchy. On the other hand, free entry independence is also intuitively preferred. With these two conditions combined, we are led uniquely to the Gaussian measure. Now the only parameters left free to choose are the two units $\mathcal{M}$ and $\mathcal{D}$. Following the spirit of anarchy, $\mathcal{D}$ should be chosen to make the neutrino Yukawa coupling of order unity,

$$
y_{\nu}=\frac{\sqrt{2}}{v} m_{D} \sim O(1)
$$

Throughout this paper, we choose $\mathcal{D}=30 \mathrm{GeV}$, which yields $y_{\nu} \sim 0.6$. Then we choose $\mathcal{M}$ to fix the next largest mass-square difference of light-neutrinos $\Delta m_{l}^{2}$ at $2.5 \times 10^{-3} \mathrm{eV}^{2}$ in accordance with the data. 


\begin{tabular}{|c|c|}
\hline right-handed neutrino & $\mathrm{U}(1)$ flavor charge \\
\hline$\nu_{R, 1}$ & 2 \\
$\nu_{R, 2}$ & 1 \\
$\nu_{R, 3}$ & 0 \\
\hline
\end{tabular}

Table 1. The U(1) flavor charge assignments for right-handed neutrinos used in this paper.

\subsection{Approximate U(1) flavor symmetry}

Our model (eq. (2.1)) is capable of generating a baryon asymmetry $\eta_{B 0}$ through thermal leptogenesis $[2,29]$. For the simplicity of analysis, we would like to focus on the scenario with two conditions:

(1) There is a hierarchy among heavy-neutrino masses $M_{1} \ll M_{2}, M_{3}$, so that the dominant contribution to $\eta_{B 0}$ is given by the decay of the lightest heavy neutrino $N_{1}$ [23].

(2) If we use $h_{i j}$ to denote the Yukawa couplings of heavy-neutrino mass eigenstates

$$
\Delta \mathcal{L} \supset-h_{i j} \epsilon^{a b} \bar{L}_{a i} H_{b}^{\dagger} N_{j},
$$

then the condition $h_{i 1} \ll 1$ for all $i=1,2,3$ would justify the neglect of annihilation process $N_{1} N_{1} \rightarrow l \bar{l}$, and also help driving the decay of $N_{1}$ out of equilibrium [23, 29]. This condition used to be taken as an assumption [29].

Both conditions above can be achieved by imposing a simple U(1) flavor charge on the right-handed neutrinos. For example, one can make charge assignments as shown in table 1. Assuming a scalar field $\phi$ carries -1 of this $U(1)$ flavor charge, one can construct neutral combinations $\nu_{\phi}$ as

$$
\nu_{\phi}=\left(\begin{array}{c}
\nu_{\phi, 1} \\
\nu_{\phi, 2} \\
\nu_{\phi, 3}
\end{array}\right)=\left(\begin{array}{c}
\nu_{R, 1} \cdot \phi^{2} \\
\nu_{R, 2} \cdot \phi \\
\nu_{R, 3} \cdot 1
\end{array}\right) .
$$

Now it only makes sense to place the random coupling matrices among these neutral combinations

$$
\Delta \mathcal{L} \supset-\bar{\nu}_{L} m_{D 0} \nu_{\phi}-\frac{1}{2} \bar{\nu}_{\phi}^{c} m_{R 0} \nu_{\phi}+\text { h.c. },
$$

where $m_{R 0}$ and $m_{D 0}$ should be generated according to Gaussian measure as in eq. (2.8)(2.9). Upon $\mathrm{U}(1)$ flavor symmetry breaking $\langle\phi\rangle=\epsilon$ with $\epsilon \simeq 0.1$, this gives

$$
\nu_{\phi} \supset\left(\begin{array}{ccc}
\epsilon^{2} & 0 & 0 \\
0 & \epsilon & 0 \\
0 & 0 & 1
\end{array}\right) \cdot \nu_{R} \equiv D_{\epsilon} \cdot \nu_{R},
$$

and hence the mass matrices

$$
\begin{aligned}
& m_{R}=D_{\epsilon} m_{R 0} D_{\epsilon}=\mathcal{M} \cdot D_{\epsilon} U_{R} D_{R} U_{R}^{T} D_{\epsilon}, \\
& m_{D}=m_{D 0} D_{\epsilon}=\mathcal{D} \cdot U_{1} D_{0} U_{2}^{\dagger} D_{\epsilon} .
\end{aligned}
$$


Let us parameterize the heavy-neutrino mass matrix as $m_{N}=U_{N} M U_{N}^{T}$, then

$$
m_{N} \approx m_{R} \sim\left(\begin{array}{ccc}
\epsilon^{4} & \epsilon^{3} & \epsilon^{2} \\
\epsilon^{3} & \epsilon^{2} & \epsilon \\
\epsilon^{2} & \epsilon & 1
\end{array}\right) \sim\left(\begin{array}{ccc}
1 & \epsilon & \epsilon^{2} \\
\epsilon & 1 & \epsilon \\
\epsilon^{2} & \epsilon & 1
\end{array}\right)\left(\begin{array}{ccc}
\epsilon^{4} & 0 & 0 \\
0 & \epsilon^{2} & 0 \\
0 & 0 & 1
\end{array}\right)\left(\begin{array}{ccc}
1 & \epsilon & \epsilon^{2} \\
\epsilon & 1 & \epsilon \\
\epsilon^{2} & \epsilon & 1
\end{array}\right)
$$

where one can identify

$$
M \sim\left(\begin{array}{ccc}
\epsilon^{4} & 0 & 0 \\
0 & \epsilon^{2} & 0 \\
0 & 0 & 1
\end{array}\right), \quad U_{N} \sim\left(\begin{array}{ccc}
1 & \epsilon & \epsilon^{2} \\
\epsilon & 1 & \epsilon \\
\epsilon^{2} & \epsilon & 1
\end{array}\right) .
$$

Clearly a hierarchy among heavy neutrino masses is achieved. Furthermore, the heavy neutrino mass eigenstates are $N=U_{N}^{T} \nu_{R}$. Since

$$
\Delta \mathcal{L} \supset-\epsilon^{a b} \bar{L}_{a} H_{b}^{\dagger} y_{\nu} \nu_{R} \equiv-h_{i j} \epsilon^{a b} \bar{L}_{a i} H_{b}^{\dagger} N_{j},
$$

we obtain the Yukawa coupling $h_{i j}$ as

$$
\begin{aligned}
h & =y_{\nu} U_{N}^{*}=\frac{\sqrt{2}}{v} m_{D} U_{N}^{*} \\
& \sim \frac{\sqrt{2}}{v} m_{D 0}\left(\begin{array}{ccc}
\epsilon^{2} & 0 & 0 \\
0 & \epsilon & 0 \\
0 & 0 & 1
\end{array}\right)\left(\begin{array}{ccc}
1 & \epsilon & \epsilon^{2} \\
\epsilon & 1 & \epsilon \\
\epsilon^{2} & \epsilon & 1
\end{array}\right) \\
& \sim \frac{\sqrt{2}}{v} m_{D 0}\left(\begin{array}{ccc}
\epsilon^{2} & \epsilon^{3} & \epsilon^{4} \\
\epsilon^{2} & \epsilon & \epsilon^{2} \\
\epsilon^{2} & \epsilon & 1
\end{array}\right) .
\end{aligned}
$$

We see that $h_{i 1} \sim \epsilon^{2} \ll 1$ is guaranteed for all $i=1,2,3$.

It is worth noting that the light-neutrino mass matrix $m_{\nu}$ is not affected by our $\mathrm{U}(1)$ flavor change assignment on right-handed neutrinos (table 1). The hierarchical matrix $D_{\epsilon}$ cancels due to the seesaw mechanism [30-35]:

$$
m_{\nu}=m_{D} m_{R}^{-1} m_{D}^{T}=m_{D 0} m_{R 0}^{-1} m_{D 0}^{T} .
$$

Therefore all properties of light neutrinos do not depend on the particular $U(1)$ flavor charge assignment nor the size of the breaking parameter $\epsilon$. The leptogenesis aspect is the only discussion in the paper where this flavor structure is relevant.

\section{Consequences}

In this section, we present our Monte Carlo results based on the sampling measure described in the last section. 


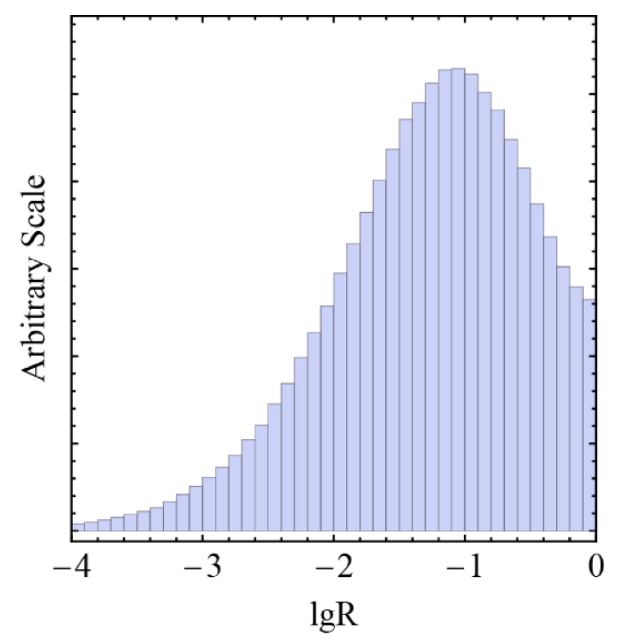

Figure 1. Histogram of $\lg R=\log _{10} R$ with $10^{6}$ occurrences collected.

\subsection{Light-neutrino mixings and mass splitting ratio}

We parameterize the light neutrino mass matrix as

$$
m_{\nu}=m_{D} m_{R}^{-1} m_{D}^{T} \equiv U_{\nu} m U_{\nu}^{T}
$$

with $U_{\nu}$ a unitary matrix and $m$ a real diagonal matrix with non-negative elements. We also follow a conventional way to label the three masses of the light neutrinos: first sort them in the ascending order $m_{11} \leq m_{22} \leq m_{33}$. Then there are two mass-squared splittings $m_{22}^{2}-m_{11}^{2}$ and $m_{33}^{2}-m_{22}^{2}$. We use $\Delta m_{s}^{2}$ and $\Delta m_{l}^{2}$ to denote the smaller and larger one respectively. If $\Delta m_{s}^{2}$ is the first one, we call this scenario "normal" and take the definitions $m_{1} \equiv m_{11}, m_{2} \equiv m_{22}, m_{3} \equiv m_{33}$. Otherwise, we call it "inverted" and take $m_{1} \equiv m_{22}, m_{2} \equiv m_{33}, m_{3} \equiv m_{11}$. The columns of the unitary matrix $U_{\nu}$ should be arranged accordingly.

Predictions on light-neutrino mixings - the distributions of mixing angles $\theta_{12}, \theta_{23}, \theta_{13}$, CP phase $\delta_{C P}$, and other physical phases $\chi_{1}, \chi_{2}$ - are certainly the same as in general study of basis independent measures [18], since $U_{\nu}$ is totally governed by the Haar measure. A statistical Kolmogorov-Smirnov test shows that the predicted mixing angle distribution is completely consistent with the experimental data [21,22].

The mass-squared splitting ratio $R \equiv \Delta m_{s}^{2} / \Delta m_{l}^{2}$ is observed to be around [11]

$$
R_{\exp } \equiv \frac{7.50 \times 10^{-5}}{2.32 \times 10^{-3}}
$$

Figure 1 shows our predicted distribution of this ratio. With a probability of $R<R_{\exp }$ being $36.1 \%$, the prediction is completely consistent with the data.

For the purpose of studying other quantities, we introduce the following Mixing-Split cuts as suggested by the experimental data [11] on light-neutrino mixings and mass-squared 


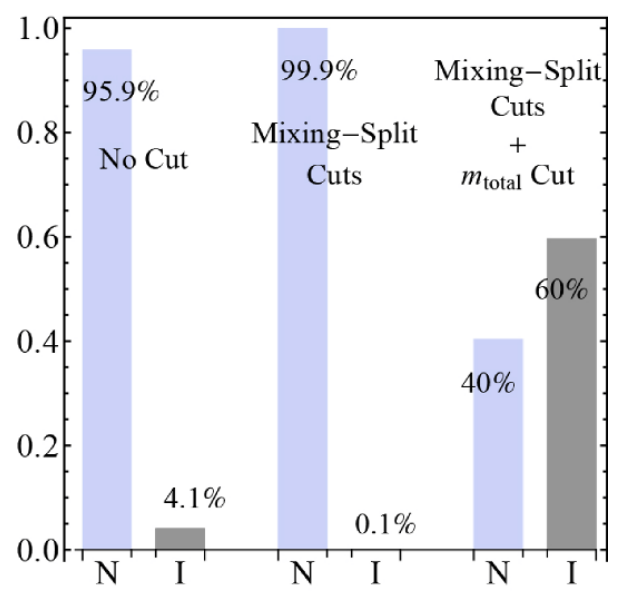

Figure 2. Fractions of normal and inverted mass hierarchy scenarios under different cuts selections, where "N" stands for normal hierarchy and "I" stands for inverted hierarchy. Each of the first two columns consists of $10^{6}$ occurrences, while the last column "Mixing-Split Cuts $+m_{\text {total }}$ Cut" contains only $10^{4}$ occurrences.

splitting ratio:

$$
\begin{aligned}
\sin ^{2} 2 \theta_{23} & =1.0 \\
\sin ^{2} 2 \theta_{12} & =0.857 \\
\sin ^{2} 2 \theta_{13} & =0.095 \\
R & \in R_{\exp } \times(1-0.05,1+0.05)
\end{aligned}
$$

\subsection{Mass hierarchy, $m_{\text {eff }}$ and $m_{\text {total }}$}

For the mass hierarchy of light-neutrino, our anarchy model predicts normal scenario being dominant. A $10^{6}$ sample Monte Carlo finds the fraction of normal scenario being $95.9 \%$ before the Mixing-Split cuts (eq. (3.3)-(3.6)), and 99.9\% after applying the cuts. Figure 2 shows the fractions of each scenario.

Neutrino anarchy allows for a random, nonzero Majorana mass matrix $m_{R}$. This means that the light neutrinos are Majorana and thus there can be neutrinoless double beta decay process. The effective mass of this process $m_{\mathrm{eff}} \equiv\left|\sum_{i} m_{i} U_{v, e i}^{2}\right|$ is definitely a very broadly interested quantity. Another quantity of general interest is the light-neutrino total mass $m_{\text {total }} \equiv m_{1}+m_{2}+m_{3}$, due to its presence in cosmological processes. Our predictions on $m_{\text {eff }}$ and $m_{\text {total }}$ are shown in figure 3 and figure 4 respectively. For each quantity, we plot both its whole distribution under Gaussian measure and its distribution after applying the Mixing-Split cuts. Distributions of $m_{\text {eff }}$ and $m_{\text {total }}$ under Gaussian measure were also studied recently in [36]. Their results are in agreement with ours. The small difference is due to the difference in taking cuts on neutrino mixing angles.

We see from figure 3 that most of the time $m_{\mathrm{eff}} \lesssim 0.05 \mathrm{eV}$. It becomes even smaller after we apply the Mixing-Split Cuts, mainly below $m_{\text {eff }} \lesssim 0.01 \mathrm{eV}$. This is very challenging to experimental sensitivity. For $m_{\text {total }}$, figure 4 shows it being predicted to be very close 

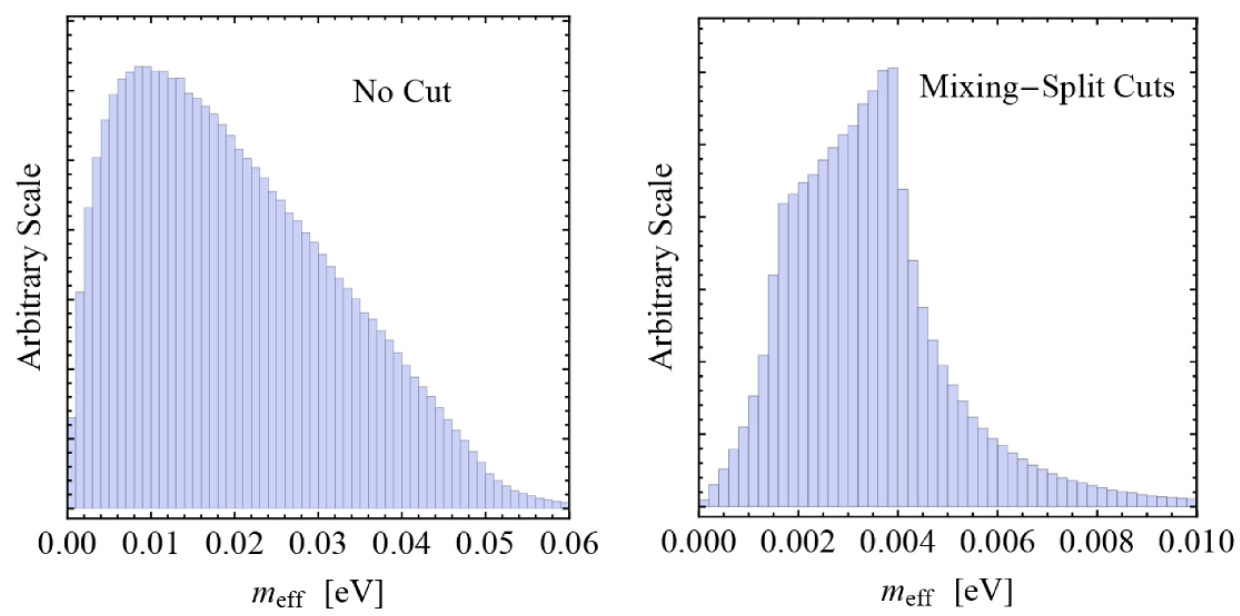

Figure 3. Histogram of $m_{\text {eff }}$ with $10^{6}$ occurrences collected. Left/Right panel shows distribution before/after applying the Mixing-Split cuts.
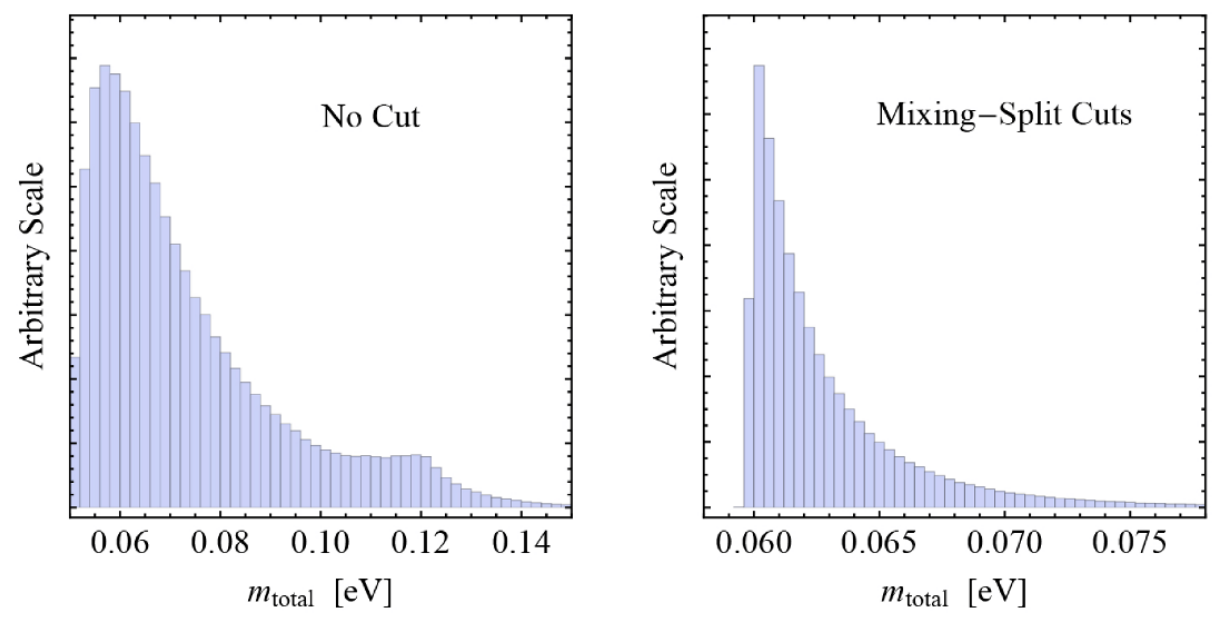

Figure 4. Histogram of $m_{\text {total }}$, with $10^{6}$ occurrences collected. Left/Right panel shows distribution before/after applying the Mixing-Split cuts.

to the current lower bound $\sim 0.06 \mathrm{eV}$. The kink near $0.1 \mathrm{eV}$ is due to the superposition of the two mass hierarchy scenarios.

To understand each component better, we show the distributions of $m_{\text {eff }}$ and $m_{\text {total }}$ in figure 5 and figure 6 for both before/after applying the cuts and normal/inverted hierarchy scenario. As figure 6 shows, under either hierarchy scenario, $m_{\text {total }}$ is likely to reside very close to its corresponding lower bound, especially after applying the cuts. Very interestingly, however, recent BOSS analysis suggests $m_{\text {total }}$ could be quite large, with a center value $\sim 0.36 \mathrm{eV}[26]$. As seen clearly from figure 6 , a large value of $m_{\text {total }}$ would favor inverted scenario. We will discuss some possible consequences of this suggestion in section 5 . 

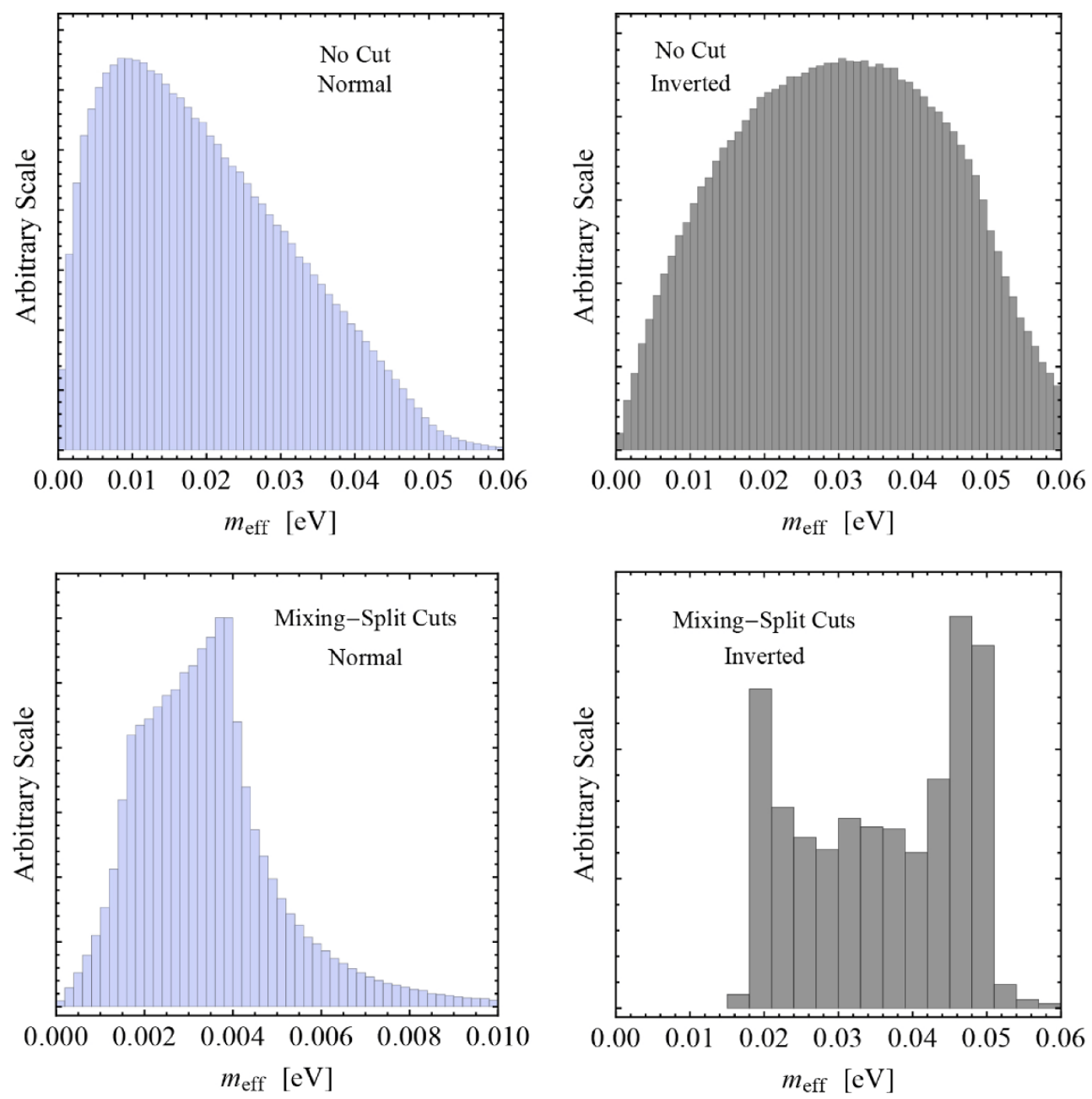

Figure 5. Histogram of $m_{\text {eff }}$ with two mass hierarchy scenarios plotted separately. Left/Right column shows distribution under normal/inverted scenario. Upper/Lower row shows distribution before/after applying the Mixing-Split cuts. The plot of inverted scenario with Mixing-Split cuts applied (right bottom) contains $10^{4}$ occurrences, while other plots contain $10^{6}$ occurrences.

\subsection{Leptogenesis}

As explained in section 2, with our approximate $\mathrm{U}(1)$ flavor symmetry, the baryon asymmetry $\eta_{B 0} \equiv \frac{n_{B}}{n_{\gamma}}$ can be computed through the standard leptogenesis calculations [23, 24]. For each event of $m_{R}$ and $m_{D}$ generated, we solve the following Boltzmann equations numerically.

$$
\begin{aligned}
\frac{d N_{1}}{d z} & =-\left(N_{1}-N_{1}^{\mathrm{eq}}\right)(D+S), \\
\frac{d N_{B-L}}{d z} & =-\left(N_{1}-N_{1}^{\mathrm{eq}}\right) \varepsilon D-N_{B-L} W,
\end{aligned}
$$

where we have followed the notations in [23] and [24].

The argument $z \equiv M_{1} / T$ is evolved from $z_{i}=0.001$ to $z_{f}=20.0$. Evolving $z$ further beyond 20.0 is not necessary, because the value of $N_{B-L}$ becomes frozen shortly after 

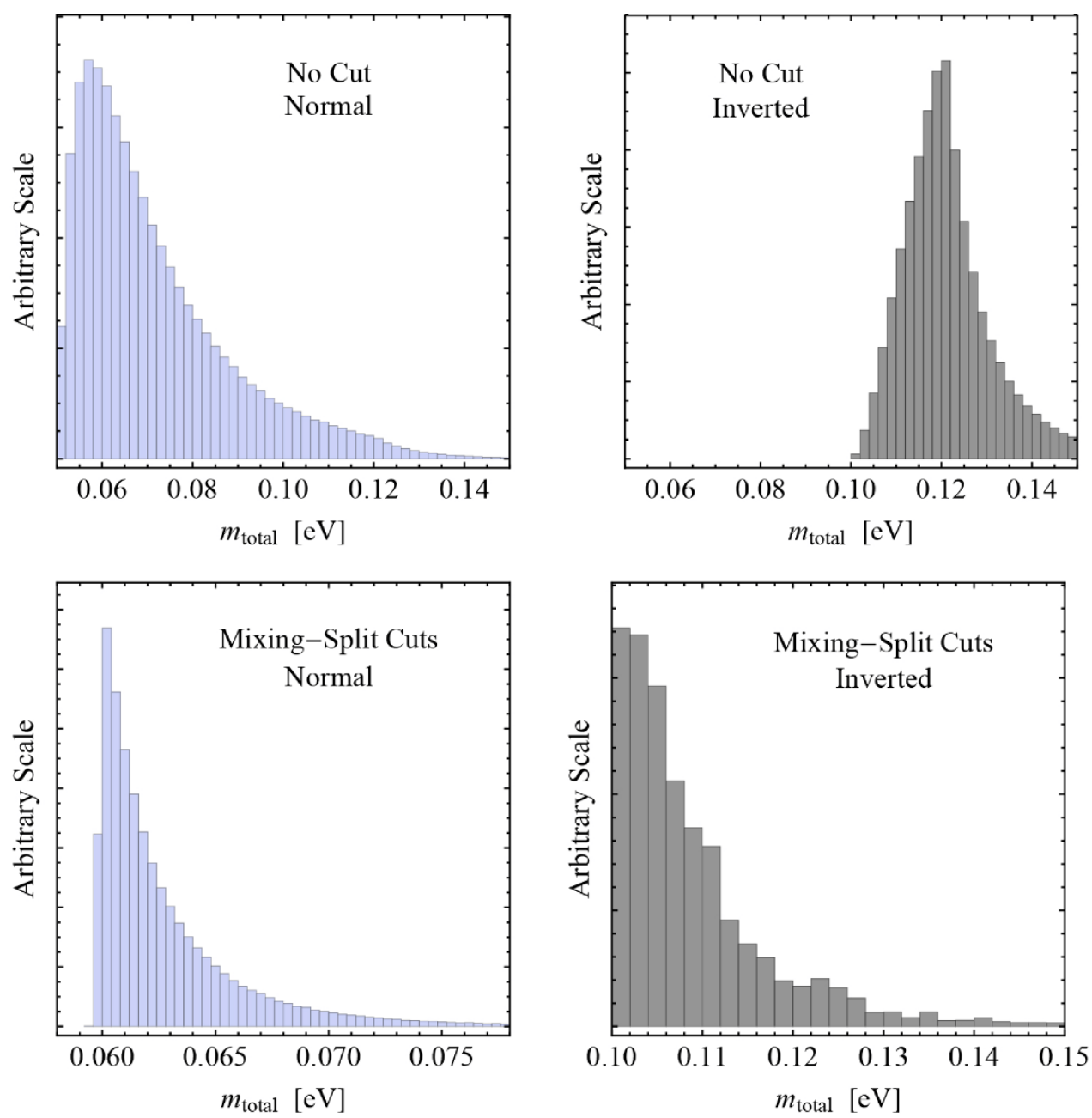

Figure 6. Histogram of $m_{\text {total }}$ with two mass hierarchy scenarios plotted separately. Left/Right column shows distribution under normal/inverted scenario. Upper/Lower row shows distribution before/after applying the Mixing-Split cuts. The plot of inverted scenario with Mixing-Split cuts applied (right bottom) contains $10^{4}$ occurrences, while other plots contain $10^{6}$ occurrences.

$z>10.0$. The baryon asymmetry is then given by $\eta_{B 0}=0.013 N_{B-L}^{0} \approx 0.013 N_{B-L}\left(z_{f}\right)[23]$. Due to randomly generated $m_{R}$ and $m_{D}$, we have equal chances for $\varepsilon$ to be positive or negative. It is the absolute value that is meaningful. We take the initial condition $N_{B-L}\left(z_{i}\right)=0$. We actually tried two typical initial conditions for $N_{1}, N_{1}\left(z_{i}\right)=0$ and $N_{1}\left(z_{i}\right)=N_{1}^{\text {eq }}\left(z_{i}\right)$, and found no recognizable differences. The distributions of $\eta_{B 0}$, both before and after applying the Mixing-Split cuts, are shown in figure 7 . We see from figure that our prediction on $\eta_{B 0}$ is about the correct order of magnitude as $\eta_{B 0, \exp } \approx 6 \times 10^{-10}$ [37].

Let us try to understand the results from some crude estimates. First, let us see why there is almost no difference between the two initial conditions $N_{1}\left(z_{i}\right)=0$ and $N_{1}\left(z_{i}\right)=$ $N_{1}^{\mathrm{eq}}\left(z_{i}\right)$. The decay function $D(z)$ has the form [24]:

$$
D(z)=\alpha_{D} \frac{K_{1}(z)}{K_{2}(z)} z
$$



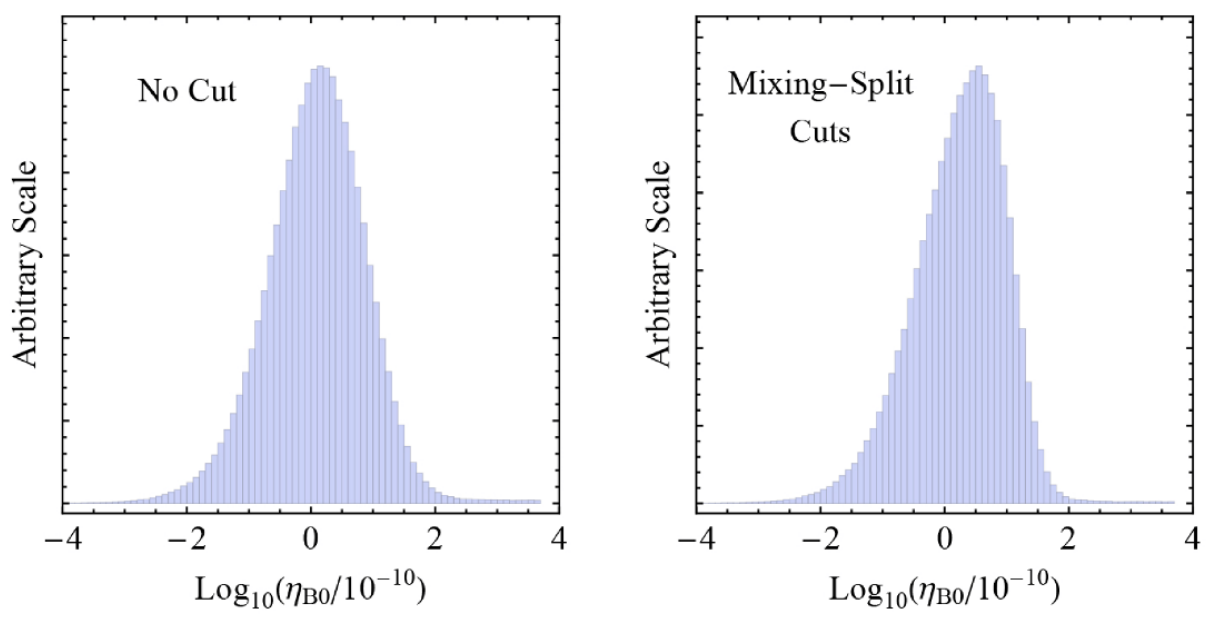

Figure 7. Histogram of $\eta_{B 0}$ with $10^{6}$ occurrences collected. Left/Right panel shows distribution before/after applying Mixing-Split cuts.

where $K_{1}(z)$ and $K_{2}(z)$ are modified Bessel functions, and the constant $\alpha_{D}$ is proportional to the effective neutrino mass $\tilde{m}_{1}$ :

$$
\alpha_{D} \propto \tilde{m}_{1} \equiv \frac{\left(m_{D}^{\dagger} m_{D}\right)_{11}}{M_{1}} .
$$

So roughly speaking, after $z \gtrsim 1$, the modified Bessel functions become rather close and $D(z)$ increases linearly with $z$. But in our prescription, the value of $D(z)+S(z)$ becomes already quite large, typically around 50 , at $z=1.0$. So $N_{1}$ is forced to be very close to $N_{1}^{\text {eq }}$ thereafter and the solution to the first differential equation (eq. (3.7)) is approximately

$$
N_{1}-N_{1}^{\mathrm{eq}}=-\frac{1}{D+S} \frac{d N_{1}}{d z} \approx-\frac{1}{D+S} \frac{d N_{1}^{\mathrm{eq}}}{d z} .
$$

Of course, this initial-condition-independent solution only holds when $D+S$ is large enough, typically for $z>1.0$. The values of $N_{1}-N_{1}^{\text {eq }}$ at $z<1.0$ certainly have a considerable dependence on $N_{1}\left(z_{i}\right)$. However, the solution to the second differential equation (eq. (3.8)) is

$$
N_{B-L}^{0}=\varepsilon \cdot \int_{0}^{\infty} d z \frac{D}{D+S} \frac{d N_{1}}{d z} e^{-\int_{z}^{\infty} W\left(z^{\prime}\right) d z^{\prime}} .
$$

And due to the shape of $W(z)$, yield of $N_{B-L}^{0}$ at $z<1.0$ is significantly suppressed by a factor $e^{-\int_{z}^{\infty} W\left(z^{\prime}\right) d z^{\prime}}$. Therefore $\eta_{B 0}$ turns out to be almost independent of $N_{1}\left(z_{i}\right)$.

Second, let us crudely estimate the order of magnitude of $\eta_{B 0}$. In addition to $D(z)$, the scattering functions $S(z)$ and washout function $W(z)$ are also proportional to $\tilde{m}_{1}$. So $\tilde{m}_{1}$ is the key factor that significantly affects the evolution of eq. (3.7) and (3.8) [24]. In our anarchy model, apart from the overall units, the mass matrix entries are $O(1)$ numbers, so we expect

$$
\tilde{m}_{1}=O(1) \cdot \frac{\mathcal{D}^{2}}{\mathcal{M}}
$$


This is just the light-neutrino mass scale. Because in our simulation, $\mathcal{M}$ is chosen such that $\Delta m_{l}^{2}=2.5 \times 10^{3} \mathrm{eV}^{2}$, we have

$$
\frac{\mathcal{D}^{2}}{\mathcal{M}}=O(1) \cdot \sqrt{\Delta m_{l}^{2}}=O(1) \cdot 0.05 \mathrm{eV}
$$

Therefore most of the time, our model is in the "strong washout regime" [24], since $\tilde{m}_{1}$ is much larger than the equilibrium neutrino mass $m_{*}$ :

$$
\tilde{m}_{1} \sim 0.05 \mathrm{eV} \gg m_{*} \sim 10^{-3} \mathrm{eV} .
$$

In this regime, the integral in eq. (3.12), which is called efficiency factor $\kappa_{f}$, should be around [24]

$$
\kappa_{f}=\int_{0}^{\infty} d z \frac{D}{D+S} \frac{d N_{1}}{d z} e^{-\int_{z}^{\infty} W\left(z^{\prime}\right) d z^{\prime}} \sim 10^{-2} .
$$

Thus our baryon asymmetry is

$$
\eta_{B 0}=0.013 N_{B-L}^{0} \sim 1.3 \times 10^{-4} \cdot \varepsilon .
$$

To estimate the CP asymmetry $\varepsilon$, we notice that (following the notation of [24])

$$
K \equiv h^{\dagger} h \sim\left(\frac{\sqrt{2}}{v} \mathcal{D}\right)^{2}\left(\begin{array}{ccc}
\epsilon^{4} & \epsilon^{3} & \epsilon^{2} \\
\epsilon^{3} & \epsilon^{2} & \epsilon \\
\epsilon^{2} & \epsilon & 1
\end{array}\right),
$$

and thus

$$
\begin{aligned}
\varepsilon & =\varepsilon_{V}+\varepsilon_{M} \sim \frac{3}{16 \pi} \sum_{k=1}^{3} \frac{\operatorname{Im}\left(K_{1 k}^{2}\right)}{K_{11}} \frac{M_{1}}{M_{k}} \\
& =\frac{3}{16 \pi}\left[\frac{\operatorname{Im}\left(K_{12}^{2}\right)}{K_{11}} \frac{M_{1}}{M_{2}}+\frac{\operatorname{Im}\left(K_{13}\right)}{K_{11}} \frac{M_{1}}{M_{3}}\right] \\
& \sim \frac{3}{16 \pi}\left(\frac{\sqrt{2}}{v} \mathcal{D}\right)^{2}\left(\frac{\epsilon^{6}}{\epsilon^{4}} \epsilon^{2}+\frac{\epsilon^{4}}{\epsilon^{4}} \epsilon^{4}\right) \\
& \sim \frac{3}{4 \pi}\left(\frac{\mathcal{D}}{v}\right)^{2} \epsilon^{4} \sim 3 \times 10^{-7} .
\end{aligned}
$$

So the baryon asymmetry is expected to be around $\eta_{B 0} \sim 1.3 \times 10^{-4} \cdot \varepsilon \sim 4 \times 10^{-11}$, multiplied by some $O(1)$ factor. This is what we see from figure 7 .

Our use of U(1) flavor symmetry breaking plays an essential role to produce the correct order of $\varepsilon\left(\varepsilon \sim \epsilon^{4}\right)$ and thus $\eta_{B 0}$. It is thus interesting to investigate what would happen if we had a different $\mathrm{U}(1)$ charge assignment. An arbitrary charge assignment would be, upon symmetry breaking, equivalent to an arbitrary choice of $D_{\epsilon}$ parameterized as

$$
D_{\epsilon}=\left(\begin{array}{ccc}
\epsilon_{1} & 0 & 0 \\
0 & \epsilon_{2} & 0 \\
0 & 0 & \epsilon_{3}
\end{array}\right)=\epsilon_{3}\left(\begin{array}{ccc}
\epsilon_{r 1} \epsilon_{r 2} & 0 & 0 \\
0 & \epsilon_{r 2} & 0 \\
0 & 0 & 1
\end{array}\right)
$$


where $\epsilon_{1}, \epsilon_{2}, \epsilon_{3} \lesssim 1$, and we have defined $\epsilon_{r 2} \equiv \epsilon_{2} / \epsilon_{3}$ and $\epsilon_{r 1} \equiv \epsilon_{1} / \epsilon_{2}$ for convenience. To make the simplest scenario of leptogenesis work, we need the hierarchy among the heavy-neutrino masses. So we restrict ourselves to the case $\epsilon_{r 1}, \epsilon_{r 2} \ll 1$.

The Majorana mass matrix now becomes

$$
\begin{aligned}
m_{R} & =D_{\epsilon} m_{R 0} D_{\epsilon} \sim \epsilon_{3}^{2}\left(\begin{array}{ccc}
\epsilon_{r 1}^{2} \epsilon_{r 2}^{2} & \epsilon_{r 1} \epsilon_{r 2}^{2} & \epsilon_{r 1} \epsilon_{r 2} \\
\epsilon_{r 1} \epsilon_{r 2}^{2} & \epsilon_{r 2}^{2} & \epsilon_{r 2} \\
\epsilon_{r 1} \epsilon_{r 2} & \epsilon_{r 2} & 1
\end{array}\right) \\
& \sim \epsilon_{3}^{2}\left(\begin{array}{ccc}
1 & \epsilon_{r 1} & \epsilon_{r 1} \epsilon_{r 2} \\
\epsilon_{r 1} & 1 & \epsilon_{r 2} \\
\epsilon_{r 1} \epsilon_{r 2} & \epsilon_{r 2} & 1
\end{array}\right) \times\left(\begin{array}{ccc}
\epsilon_{r 1}^{2} \epsilon_{r 2}^{2} & 0 & 0 \\
0 & \epsilon_{r 2}^{2} & 0 \\
0 & 0 & 1
\end{array}\right)\left(\begin{array}{ccc}
1 & \epsilon_{r 1} & \epsilon_{r 1} \epsilon_{r 2} \\
\epsilon_{r 1} & 1 & \epsilon_{r 2} \\
\epsilon_{r 1} \epsilon_{r 2} & \epsilon_{r 2} & 1
\end{array}\right),
\end{aligned}
$$

which gives

$$
\begin{gathered}
M \sim \epsilon_{3}^{2}\left(\begin{array}{ccc}
\epsilon_{r 1}^{2} \epsilon_{r 2}^{2} & 0 & 0 \\
0 & \epsilon_{r 2}^{2} & 0 \\
0 & 0 & 1
\end{array}\right), \\
U_{N} \sim\left(\begin{array}{ccc}
1 & \epsilon_{r 1} & \epsilon_{r 1} \epsilon_{r 2} \\
\epsilon_{r 1} & 1 & \epsilon_{r 2} \\
\epsilon_{r 1} \epsilon_{r 2} & \epsilon_{r 2} & 1
\end{array}\right) .
\end{gathered}
$$

The Dirac mass matrix becomes

$$
m_{D}=m_{D 0} D_{\epsilon},
$$

which gives the Yukawa coupling $h$ and $K \equiv h^{\dagger} h$ as

$$
\begin{aligned}
h & =\frac{\sqrt{2}}{v} m_{D} U_{N}{ }^{*} \\
& \sim \frac{\sqrt{2}}{v} m_{D 0} \epsilon_{3}\left(\begin{array}{ccc}
\epsilon_{r 1} \epsilon_{r 2} & \epsilon_{r 1}^{2} \epsilon_{r 2} & \epsilon_{r 1}^{2} \epsilon_{r 2}^{2} \\
\epsilon_{r 1} \epsilon_{r 2} & \epsilon_{r 2} & \epsilon_{r 2}^{2} \\
\epsilon_{r 1} \epsilon_{r 2} & \epsilon_{r 2} & 1
\end{array}\right) \\
K & \sim\left(\frac{\sqrt{2}}{v} \mathcal{D}\right)^{2} \epsilon_{3}^{2}\left(\begin{array}{ccc}
\epsilon_{r 1}^{2} \epsilon_{r 2}^{2} & \epsilon_{r 1} \epsilon_{r 2}^{2} & \epsilon_{r 1} \epsilon_{r 2} \\
\epsilon_{r 1} \epsilon_{r 2}^{2} & \epsilon_{r 2}^{2} & \epsilon_{r 2} \\
\epsilon_{r 1} \epsilon_{r 2} & \epsilon_{r 2} & 1
\end{array}\right) .
\end{aligned}
$$

So our $\varepsilon$ is given by

$$
\begin{aligned}
\varepsilon & =\varepsilon_{V}+\varepsilon_{M} \sim \frac{3}{16 \pi} \sum_{k=1}^{3} \frac{\operatorname{Im}\left(K_{1 k}^{2}\right)}{K_{11}} \frac{M_{1}}{M_{k}} \\
& \sim \frac{3}{8 \pi}\left(\frac{\sqrt{2}}{v} \mathcal{D}\right)^{2} \epsilon_{3}^{2} \epsilon_{r 1}^{2} \epsilon_{r 2}^{2} \\
& \sim \frac{3}{4 \pi}\left(\frac{\mathcal{D}}{v}\right)^{2} \epsilon_{1}^{2} .
\end{aligned}
$$

We see that under the condition $\epsilon_{r 1}, \epsilon_{r 2} \ll 1, \varepsilon$ is only sensitive to the value of $\epsilon_{1}$. 
On the other hand, the value of $\tilde{m}_{1}$ is not affected by changing $\mathrm{U}(1)$ flavor charge assignments:

$$
\begin{aligned}
\tilde{m}_{1} & \equiv \frac{\left(m_{D}^{\dagger} m_{D}\right)_{11}}{M_{1}} \sim \frac{\left(m_{D 0}^{\dagger} m_{D 0}\right)_{11}}{\left(M_{1}\right)_{0}} \frac{\epsilon_{1}^{2}}{\epsilon_{1}^{2}} \\
& =\frac{\left(m_{D 0}^{\dagger} m_{D 0}\right)_{11}}{\left(M_{1}\right)_{0}}=\left(\tilde{m}_{1}\right)_{0} .
\end{aligned}
$$

Here a subscript " 0 " is used to denote the value when there is no $\mathrm{U}(1)$ flavor charge assignment, as we did in eq. (2.13). So the strong washout condition (eq. (3.15)) still holds, and we are again led to eq. (3.17). Therefore, the baryon asymmetry $\eta_{B 0}$ can only be affected through $\varepsilon$, which in turn is only sensitive to $\epsilon_{1}$, under the condition $\epsilon_{r 1}, \epsilon_{r 2} \ll 1$.

\section{Correlations between $\eta_{B 0}$ and light-neutrino parameters}

As we can see from figure 7, the baryon asymmetry is slightly enhanced after applying the Mixing-Split cuts eq. (3.3)-(3.6). This indicates some correlation between $\eta_{B 0}$ and lightneutrino parameters. To understand this better, we would like to systematically investigate the correlations between $\eta_{B 0}$ and the light-neutrino mass matrix $m_{\nu}=U_{\nu} m U_{\nu}^{T}{ }^{2}{ }^{2}$

Although both of $\eta_{B 0}$ and $m_{\nu}$ seem to depend on the random inputs $m_{R}$ and $m_{D}$ in a complicated way, it is not hard to see that there should be no correlation between $\eta_{B 0}$ and $U_{\nu}$ (This was also pointed out in [25]). Recall that we parametrize $m_{R}$ and $m_{D}$ as in eq. (2.2)-(2.3). And due to the decomposition eq. (2.4)-(2.5), there are five independent random matrices: $U_{1}, U_{2}, U_{R}, D_{0}$ and $D_{R}$. The first thing to observe is that changing $U_{1}$ with the other four matrices fixed will not affect $\eta_{B 0}$. This is because:

1. The baryon asymmetry $\eta_{B 0}$ we have been computing in this paper is the total baryon asymmetry, including all the three generations. So $m_{D}$ enters the calculation of leptogenesis only through the form of the matrix

$$
K \equiv h^{\dagger} h=\left(\frac{\sqrt{2}}{v}\right)^{2} U_{N}^{T} m_{D}^{\dagger} m_{D} U_{N}^{*},
$$

with $m_{D}=\mathcal{D} \cdot U_{1} D_{0} U_{2}^{\dagger}$. Obviously $U_{1}$ cancels in $K$.

2. Throughout the simulation, we are also applying a built-in cut $\Delta m_{l}^{2}=2.5 \times 10^{-3} \mathrm{eV}^{2}$ by choosing the value of $\mathcal{M}$ to force it. Due to this cut, $m_{D}$ can potentially affect $\eta_{B 0}$ through the value of $\mathcal{M}$. However, since the actual relation is

$$
\begin{aligned}
m_{\nu} & =m_{D} m_{R}^{-1} m_{D}^{T} \\
& =\frac{\mathcal{D}^{2}}{\mathcal{M}} U_{1} D_{0} U_{2}^{\dagger} m_{R}^{-1} U_{2}^{*} D_{0} U_{1}^{T} \\
& =U_{\nu} m U_{\nu}^{T},
\end{aligned}
$$

\footnotetext{
${ }^{2}$ The correlation between leptogenesis and light-neutrino parameters has been recently studied in [25]. They have some overlap with our results.
} 
we see that changing $U_{1}$ would only affect $U_{\nu}$, not $m$. So no further adjustment of $\mathcal{M}$ is needed when we change $U_{1}$.

The second point to observe is that any change in $U_{\nu}$ can be achieved by a left translation

$$
U_{\nu a} \rightarrow U_{\nu b}=\left(U_{\nu b} U_{\nu a}^{-1}\right) U_{\nu a} \equiv U_{L} U_{\nu a}
$$

This in turn, can be accounted for by just a left translation in $U_{1}: U_{1 a} \rightarrow U_{1 b}=U_{L} U_{1 a}$, with the other four random matrices unchanged (see eq. (4.2)). This left translation in $U_{1}$ is thus a one-to-one mapping between the sub-sample generating $U_{\nu a}$ and the sub-sample generating $U_{\nu b}=U_{L} U_{\nu a}$. Any two events connected through this one-to-one mapping generate the same value of $\eta_{B 0}$, because changing $U_{1}$ does not change $\eta_{B 0}$. In addition, the two events have the same chance to appear, because the measure over $U_{1}$ is the Haar measure, which is invariant under the left translation. Thus the sub-sample with $U_{\nu}=U_{\nu a}$ and $U_{\nu}=U_{\nu b}$, for any arbitrary $U_{\nu a}$ and $U_{\nu b}$, will give the same distribution of $\eta_{B 0}$, namely that $\eta_{B 0}$ is independent of $U_{\nu}$. So immediately we conclude that $\eta_{B 0}$ cannot be correlated with the three mixing angles $\theta_{12}, \theta_{23}, \theta_{13}$, the $\mathrm{CP}$ phase $\delta_{C P}$, or the phases $\chi_{1}, \chi_{2}$.

Three of the Mixing-Split cuts applied to $\eta_{B 0}$ are cuts on mixing angles which we just showed not correlated with $\eta_{B 0}$. So clearly, the enhancement of $\eta_{B 0}$ is due to its non-zero correlation with $R$. To study more detail about the correlation between $\eta_{B 0}$ and the light-neutrino masses $m$, we apply a $\chi^{2}$ test of independence numerically to the joint distribution between $\eta_{B 0}$ and quantities related to $m$, including $\lg R, m_{\mathrm{eff}}$ and $m_{\text {total }}$. For each quantity with $\eta_{B 0}$, we construct a discrete joint distribution by counting the number of occurrences $O_{i j}(i, j=1, \ldots, 10)$ in an appropriate $10 \times 10$ partitioning grid. Then we obtain the expected number of occurrences $E_{i j}$ as

$$
E_{i j}=\frac{1}{n}\left(\sum_{c=1}^{10} O_{i c}\right)\left(\sum_{r=1}^{10} O_{r j}\right),
$$

where $n$ is the total number of occurrences in all $10 \times 10$ partitions. If the two random variables in question were independent of each other, we would have the test statistic

$$
X=\sum_{i, j=1}^{10} \frac{\left(O_{i j}-E_{i j}\right)^{2}}{E_{i j}},
$$

satisfying the $\chi^{2}$ distribution with degrees of freedom $(10-1) \times(10-1)=81$. We then compute the probability $P\left(\chi^{2}>X\right)$ for the hypothesis distribution $\chi^{2}(81)$ to see if the independence hypothesis is likely. Our results from a $n=3,000,000$ sample Monte Carlo are shown in table 2 .

Unambiguously, $\eta_{B 0}$ has nonzero correlations with $\lg R, m_{\mathrm{eff}}$, and $m_{\mathrm{total}}$. To see the tendency of the correlations, we draw scatter plots with 5,000 occurrences (figure 8). The plots show that all the three quantities are negatively correlated with $\eta_{B 0}$. For example the left panel of figure 8 tells us that a smaller $\lg R$ would favor a larger $\eta_{B 0}$. This explains the slight enhancement of $\eta_{B 0}$ after applying Mixing-Split cuts. But as the scatter plots show, the correlations are rather weak. 


\begin{tabular}{|c|c|c|}
\hline & $X$ & $P\left(\chi^{2}>X\right)$ \\
\hline $\lg R$ & $1.43 \times 10^{5}$ & $3.81 \times 10^{-30908}$ \\
$m_{\mathrm{eff}}$ & $8.06 \times 10^{3}$ & $1.24 \times 10^{-1655}$ \\
$m_{\text {total }}$ & $1.04 \times 10^{5}$ & $7.24 \times 10^{-22445}$ \\
\hline
\end{tabular}

Table 2. $\chi^{2}$ test of independence between $\eta_{B 0}$ and $\lg R, m_{\mathrm{eff}}, m_{\mathrm{total}}$.
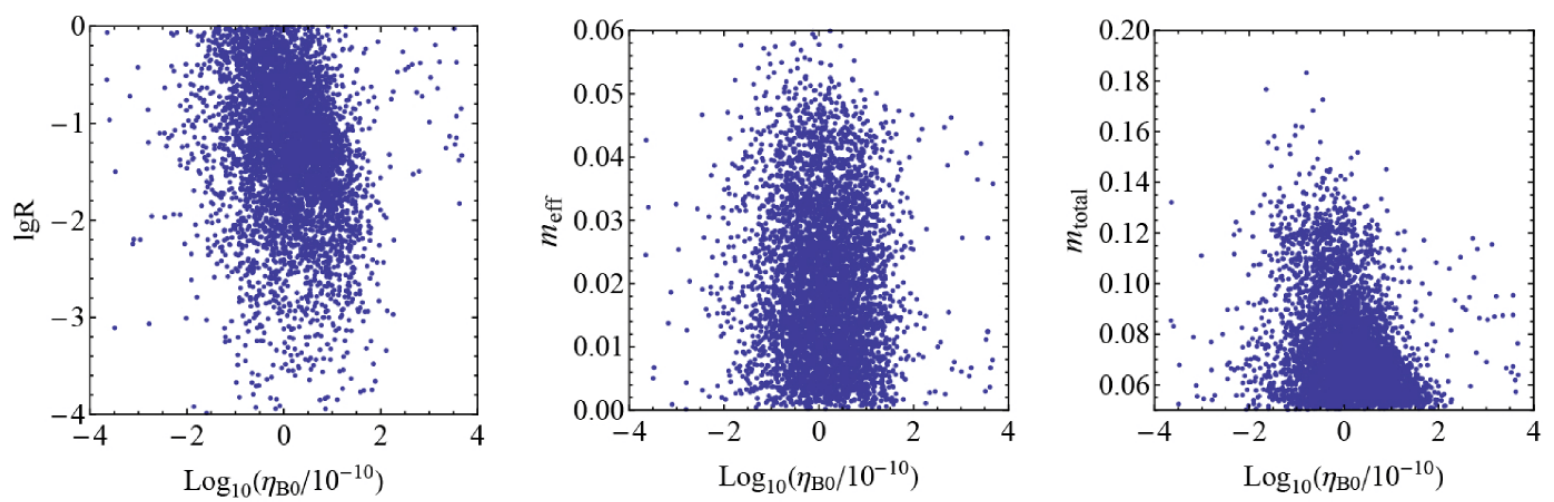

Figure 8. Scatter plots for $\eta_{B 0}$ with $\lg R, m_{\text {eff }}$, and $m_{\text {total }}$. Each plot shows a sample of 5,000 occurrences.

\section{Possible consequences of a large $\boldsymbol{m}_{\text {total }}$}

As mentioned previously, a recent BOSS analysis suggests $m_{\text {total }}$ possibly quite large, $m_{\text {total }}=0.36 \pm 0.10 \mathrm{eV}[26]$. Currently their uncertainty is still large, and thus no conclusive argument can be made. If in future the uncertainty pins down near its current central value, anarchy prediction (figure 4) would be obviously inconsistent with it and becomes ruled out. On the other hand, if the central value also comes down significantly, it could still be well consistent with anarchy prediction.

Without the knowledge of future data, we would like to answer the following question: assuming the future data be consistent with anarchy, could a relatively large $m_{\text {total }}$ dramatically change anarchy's predictions on other quantities? For this purpose, we introduce a heuristic $m_{\text {total }}$ cut:

$$
m_{\text {total }}>0.1 \mathrm{eV},
$$

just to get a sense of how much our predictions could be changed if there turns out to be a large but still consistent $m_{\text {total }}$.

We collect $10^{4}$ occurrences that pass both the Mixing-Split cuts and the $m_{\text {total }}$ cut. It turns out that the predictions change quite significantly. We see from figure 2 that the mass hierarchy prediction is overturned, with normal hierarchy only $40 \%$ and inverted scenario more likely. This can be expected from figure 6 . The predictions of $m_{\text {eff }}$ and $\eta_{B 0}$ are shown in figure 9 . We see that $m_{\text {eff }}$ exhibits a very interesting bipolar distribution. Its overall expectation value also becomes about an order of magnitude larger than before and thus much less challenging to the neutrinoless double beta decay experiments. The prediction 

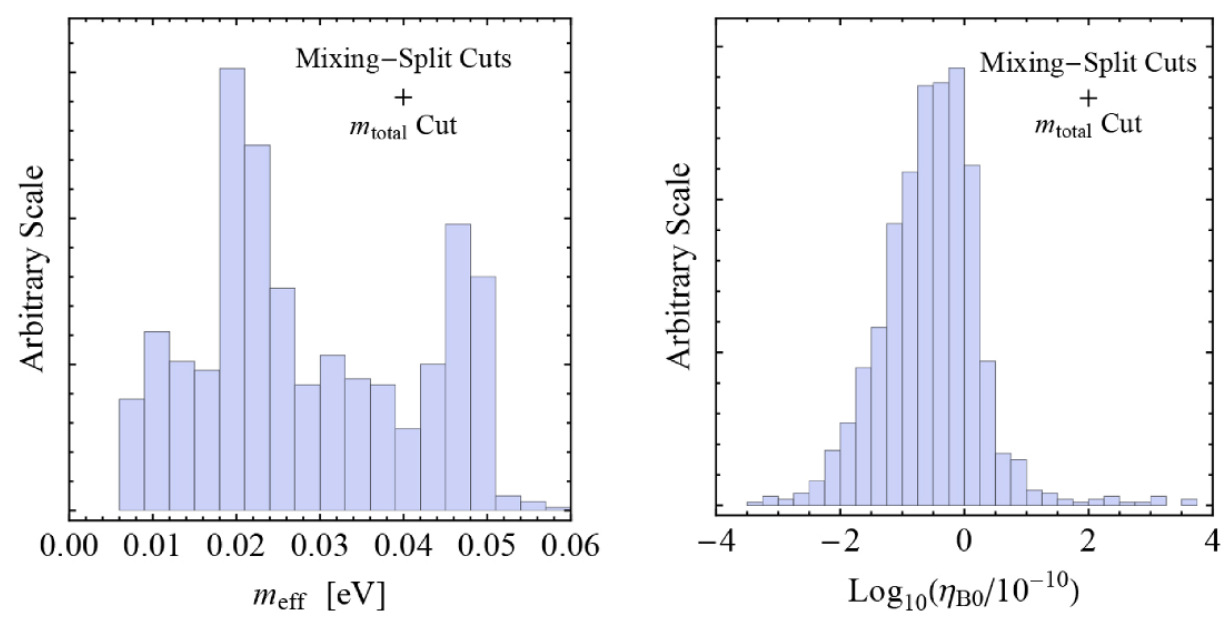

Figure 9. Histograms of $m_{\text {eff }}$ (left) and $\eta_{B 0}$ (right) with $10^{4}$ occurrences passed both Mixing-Split cuts and the $m_{\text {total }}$ cut.

on $\eta_{B 0}$ drops by about an order of magnitude, but the observed baryon asymmetry is still very likely to be achieved.

\section{Conclusions}

We have shown that basis independence and free entry independence lead uniquely to Gaussian measure for $m_{R}$ and $m_{D}$. We also showed that an approximate U(1) flavor symmetry can make leptogenesis feasible for neutrino anarchy. Combining the two, we find anarchy model successfully generate the observed amount of baryon asymmetry. Same sampling model is used to study other quantities related to neutrino masses. We found the chance of normal mass hierarchy is as high as $99.9 \%$. The effective mass of neutrinoless double beta decay $m_{\text {eff }}$ would probably be well beyond the current experimental sensitivity. The neutrino total mass $m_{\text {total }}$ is a little more optimistic. Correlations between baryon asymmetry and light-neutrino quantities were also investigated. We found $\eta_{B 0}$ not correlated with light-neutrino mixings or phases, but weakly correlated with $R, m_{\text {eff }}$, and $m_{\text {total }}$, all with negative correlation. Possible implications of recent BOSS analysis result have been discussed.

\section{Acknowledgments}

This work was supported by the U.S. DOE under Contract DE-AC02-05CH11231, and by the NSF under grants PHY-1002399 and PHY-1316783. HM was also supported by the JSPS Grant-in-Aid for Scientific Research (C) (No. 26400241), Scientific Research on Innovative Areas (No. 26105507), and by WPI, MEXT, Japan. 


\section{A Basis independence and free entry independence uniquely lead us to Gaussian measure}

Let us abstractly write all choices of measure in the form

$$
d m=\left(\prod_{i j} d m_{i j}\right) \cdot e^{-f\left(\left\{m_{i j}\right\}\right)},
$$

where $m$ stands for $m_{R}$ or $m_{D}, \prod_{i j}$ and $\left\{m_{i j}\right\}$ run over all the free entries of $m$. We want the form of $f\left(\left\{m_{i j}\right\}\right)$ so that the measure above has both basis independence and independence among $m_{i j}$.

Let us first consider $m_{D}$. For $N \times N m_{D}$, there are $N^{2}$ free entries: $m_{11}, m_{12}, \ldots, m_{N N}$. For convenience, let us rename them as $x_{1}, x_{2}, \ldots, x_{n}$, where $n=N^{2}$. Then $\left\{x_{i}\right\}$ forms an irreducible unitary representation of the basis transformation group $\mathrm{U}(3)_{L} \times \mathrm{U}(3)_{R}$ in flavor space:

$$
\begin{aligned}
m_{D} & \rightarrow m_{D}^{\prime}=U_{L} m_{D} U_{R}^{\dagger}, \\
x & \rightarrow x^{\prime}=\Lambda x,
\end{aligned}
$$

where $\Lambda=U_{L} \otimes U_{R}^{*}$ is obviously unitary.

Independence of $\left\{m_{i j}\right\}$, namely $\left\{x_{i}\right\}$, requires $f\left(\left\{m_{i j}\right\}\right)$ having the form

$$
f\left(\left\{x_{i}\right\}\right)=f_{1}\left(x_{1}\right)+f_{2}\left(x_{2}\right)+\cdots+f_{n}\left(x_{n}\right) .
$$

Here since $x_{i}$ are complex arguments, all the functions $f_{i}\left(x_{i}\right)$ are actually abbreviations of $f_{i}\left(x_{i}, x_{i}^{*}\right)$. Under the transformation of eq. (A.3), basis independence requires

$$
f_{1}\left(x_{1}^{\prime}\right)+\cdots+f_{n}\left(x_{n}^{\prime}\right)=f_{1}\left(x_{1}\right)+\cdots+f_{n}\left(x_{n}\right) .
$$

Taking a derivative with respect to $x_{i}^{*}$ yields

$$
\sum_{j} \Lambda_{j i}^{*} \frac{\partial f_{j}\left(x_{j}^{\prime}\right)}{\partial x_{j}^{\prime *}}=\frac{\partial f_{i}\left(x_{i}\right)}{\partial x_{i}^{*}} .
$$

Since $\Lambda$ is unitary, this is the same as

$$
\frac{\partial f_{i}\left(x_{i}^{\prime}\right)}{\partial x_{i}^{\prime *}}=\sum_{j} \Lambda_{i j} \frac{\partial f_{j}\left(x_{j}\right)}{\partial x_{j}^{*}} .
$$

Thus $\frac{\partial f_{i}\left(x_{i}\right)}{\partial x_{i}^{*}}$ transform in the same way as $x_{i}$. Because $x_{i}$ forms an irreducible representation of the transformation eq. (A.3), the only possibility for $\frac{\partial f_{i}\left(x_{i}\right)}{\partial x_{i}^{*}}$ is

$$
\left(\begin{array}{c}
\frac{\partial f_{1}\left(x_{1}\right)}{\partial x_{1}^{*}} \\
\vdots \\
\frac{\partial f_{n}\left(x_{n}\right)}{\partial x_{n}^{*}}
\end{array}\right)=c_{a}\left(\begin{array}{c}
x_{1} \\
\vdots \\
x_{n}
\end{array}\right)
$$


with $c_{a}$ an arbitrary constant. Similarly, taking a derivative of eq. (A.5) with respect to $x_{i}$ will give us

$$
\left(\begin{array}{c}
\frac{\partial f_{1}\left(x_{1}\right)}{\partial x_{1}} \\
\vdots \\
\frac{\partial f_{n}\left(x_{n}\right)}{\partial x_{n}}
\end{array}\right)=c_{b}\left(\begin{array}{c}
x_{1}^{*} \\
\vdots \\
x_{n}^{*}
\end{array}\right) .
$$

Combining eq. (A.8) and (A.9) we get

$$
\begin{aligned}
f\left(\left\{m_{D, i j}\right\}\right) & =c_{1}\left(x_{1} x_{1}^{*}+\cdots+x_{n} x_{n}^{*}\right)+c_{2} \\
& =c_{1}\left(\sum_{i j}\left|m_{D, i j}\right|^{2}\right)+c_{2} .
\end{aligned}
$$

An important condition in this proof is that $x$ forms an irreducible unitary representation of the basis transformation group, $\mathrm{U}(3)_{L} \times \mathrm{U}(3)_{R}$ in the case of $m_{D}$. For the case of $m_{R}$, this condition still holds. The relevant basis transformation group for $m_{R}$ is just $\mathrm{U}(3)_{R}$

$$
\begin{aligned}
m_{R} & \rightarrow m_{R}^{\prime}=U_{R} m_{R} U_{R}^{T}, \\
x & \rightarrow x^{\prime}=\Lambda x .
\end{aligned}
$$

$\Lambda=U_{R} \otimes U_{R}$ is reducible in general: $3 \otimes 3=6 \oplus 3$, but our $m_{R}$ is symmetric by definition, which only forms the irreducible subspace "6" (Note that if $m_{R}$ were real symmetric, this symmetric subspace "6" would be further reducible.). So same as in eq. (A.10), we get

$$
\begin{aligned}
f\left(\left\{m_{R, i j}\right\}\right) & =c_{1}\left(x_{1} x_{1}^{*}+\cdots+x_{n} x_{n}^{*}\right)+c_{2} \\
& =c_{1}\left(\sum_{i}\left|m_{R, i i}\right|^{2}+2 \sum_{i<j}\left|m_{R, i j}\right|^{2}\right)+c_{2} .
\end{aligned}
$$

In eq. (A.10) and (A.13), $c_{1}$ corresponds to the freedom of adjusting $\mathcal{D}$ and $\mathcal{M}$, while $c_{2}$ is just an overall normalization factor. Plugging them back into eq. (A.1), we get the Gaussian measure of $m_{D}$ and $m_{R}$ as in eq. (2.8) and (2.9).

Open Access. This article is distributed under the terms of the Creative Commons Attribution License (CC-BY 4.0), which permits any use, distribution and reproduction in any medium, provided the original author(s) and source are credited.

\section{References}

[1] S. Tremaine and J.E. Gunn, Dynamical Role of Light Neutral Leptons in Cosmology, Phys. Rev. Lett. 42 (1979) 407 [INSPIRE].

[2] M. Fukugita and T. Yanagida, Baryogenesis Without Grand Unification, Phys. Lett. B 174 (1986) 45 [INSPIRE].

[3] H. Murayama, H. Suzuki, T. Yanagida and J. Yokoyama, Chaotic inflation and baryogenesis in supergravity, Phys. Rev. D 50 (1994) 2356 [hep-ph/9311326] [INSPIRE]. 
[4] H. Murayama, K. Nakayama, F. Takahashi and T.T. Yanagida, Sneutrino Chaotic Inflation and Landscape, arXiv: 1404.3857 [INSPIRE].

[5] Super-Kamiokande collaboration, Y. Fukuda et al., Evidence for oscillation of atmospheric neutrinos, Phys. Rev. Lett. 81 (1998) 1562 [hep-ex/9807003] [INSPIRE].

[6] SNO collaboration, Q.R. Ahmad et al., Direct evidence for neutrino flavor transformation from neutral current interactions in the Sudbury Neutrino Observatory, Phys. Rev. Lett. 89 (2002) 011301 [nucl-ex/0204008] [INSPIRE].

[7] KAmLAND collaboration, K. Eguchi et al., First results from KamLAND: Evidence for reactor anti-neutrino disappearance, Phys. Rev. Lett. 90 (2003) 021802 [hep-ex/0212021] [INSPIRE].

[8] DAYA-BAY collaboration, F.P. An et al., Observation of electron-antineutrino disappearance at Daya Bay, Phys. Rev. Lett. 108 (2012) 171803 [arXiv:1203.1669] [INSPIRE].

[9] RENO collaboration, J.K. Ahn et al., Observation of Reactor Electron Antineutrino Disappearance in the RENO Experiment, Phys. Rev. Lett. 108 (2012) 191802 [arXiv: 1204.0626] [INSPIRE].

[10] T2K collaboration, K. Abe et al., Observation of Electron Neutrino Appearance in a Muon Neutrino Beam, Phys. Rev. Lett. 112 (2014) 061802 [arXiv:1311.4750] [INSPIRE].

[11] Particle Data Group collaboration, J. Beringer et al., Review of Particle Physics (RPP), Phys. Rev. D 86 (2012) 010001 [INSPIRE].

[12] C.H. Albright and S.M. Barr, Bimaximal mixing in an $\mathrm{SO}(10)$ minimal Higgs model, Phys. Lett. B 461 (1999) 218 [hep-ph/9906297] [inSPIRE].

[13] G. Altarelli and F. Feruglio, Neutrino mass textures from oscillations with maximal mixing, Phys. Lett. B 439 (1998) 112 [hep-ph/9807353] [INSPIRE].

[14] R. Barbieri, L.J. Hall and A. Strumia, Textures for atmospheric and solar neutrino oscillations, Phys. Lett. B 445 (1999) 407 [hep-ph/9808333] [INSPIRE].

[15] T. Blazek, S. Raby and K. Tobe, Neutrino oscillations in a predictive SUSY GUT, Phys. Rev. D 60 (1999) 113001 [hep-ph/9903340] [INSPIRE].

[16] Y. Nomura and T. Yanagida, Bimaximal neutrino mixing in $\mathrm{SO}(10)(G U T)$, Phys. Rev. D 59 (1999) 017303 [hep-ph/9807325] [INSPIRE].

[17] L.J. Hall, H. Murayama and N. Weiner, Neutrino mass anarchy, Phys. Rev. Lett. 84 (2000) 2572 [hep-ph/9911341] [INSPIRE].

[18] N. Haba and H. Murayama, Anarchy and hierarchy, Phys. Rev. D 63 (2001) 053010 [hep-ph/0009174] [INSPIRE].

[19] C.D. Froggatt and H.B. Nielsen, Hierarchy of Quark Masses, Cabibbo Angles and CP-violation, Nucl. Phys. B 147 (1979) 277 [INSPIRE].

[20] M. Leurer, Y. Nir and N. Seiberg, Mass matrix models, Nucl. Phys. B 398 (1993) 319 [hep-ph/9212278] [INSPIRE].

[21] A. de Gouvêa and H. Murayama, Statistical test of anarchy, Phys. Lett. B 573 (2003) 94 [hep-ph/0301050] [INSPIRE].

[22] A. de Gouvêa and H. Murayama, Neutrino Mixing Anarchy: Alive and Kicking, arXiv: 1204.1249 [INSPIRE]. 
[23] W. Buchmüller, P. Di Bari and M. Plümacher, Cosmic microwave background, matter-antimatter asymmetry and neutrino masses, Nucl. Phys. B 643 (2002) 367 [Erratum ibid. B 793 (2008) 362] [hep-ph/0205349] [INSPIRE].

[24] W. Buchmüller, P. Di Bari and M. Plümacher, Leptogenesis for pedestrians, Annals Phys. 315 (2005) 305 [hep-ph/0401240] [INSPIRE].

[25] K.S. Jeong and F. Takahashi, Anarchy and Leptogenesis, JHEP 07 (2012) 170 [arXiv: 1204.5453] [INSPIRE].

[26] BOSS collaboration, F. Beutler et al., The clustering of galaxies in the SDSS-III Baryon Oscillation Spectroscopic Survey: Signs of neutrino mass in current cosmological datasets, arXiv: 1403.4599 [INSPIRE].

[27] M.L. Mehta, Random matrices. Vol. 142, Academic press (2004).

[28] Y. Bai and G. Torroba, Large-N (=3) Neutrinos and Random Matrix Theory, JHEP 12 (2012) 026 [arXiv:1210.2394] [INSPIRE].

[29] M.A. Luty, Baryogenesis via leptogenesis, Phys. Rev. D 45 (1992) 455 [InSPIRE].

[30] P. Minkowski, $\mu-->$ er at a Rate of One Out of 1-Billion Muon Decays?, Phys. Lett. B 67 (1977) 421 [INSPIRE].

[31] T. Yanagida, Horizontal Symmetry and Masses of Neutrinos, Proceedings of Workshop on the Unified Theories and the Baryon Number in the Universe, Tsukuba, Japan, February 13-14 1979, Conf. Proc. C7902131 (1979) 95.

[32] M. Gell-Mann, P. Ramond and R. Slansky, Complex Spinors and Unified Theories, Conf. Proc. C 790927 (1979) 315 [arXiv: 1306.4669] [INSPIRE].

[33] T. Yanagida, Horizontal Symmetry and Masses of Neutrinos, Prog. Theor. Phys. 64 (1980) 1103 [INSPIRE].

[34] S. Glashow, The Future of Elementary Particle Physics, NATO Adv. Study Inst. Ser. B Phys. 59 (1980) 687.

[35] R.N. Mohapatra and G. Senjanović, Neutrino Mass and Spontaneous Parity Violation, Phys. Rev. Lett. 44 (1980) 912 [INSPIRE].

[36] J. Bergstrom, D. Meloni and L. Merlo, A Bayesian comparison of U(1) lepton flavour models, Phys. Rev. D 89 (2014) 093021 [arXiv:1403.4528] [InSPIRE].

[37] WMAP collaboration, E. Komatsu et al., Seven-Year Wilkinson Microwave Anisotropy Probe (WMAP) Observations: Cosmological Interpretation, Astrophys. J. Suppl. 192 (2011) 18 [arXiv: 1001.4538$]$ [INSPIRE]. 